\title{
FAMILIAS NUMEROSAS E IRA EN DEPORTISTAS Y PRACTICANTES DE ACTIVIDAD FÍSICA
}

\author{
NUMEROUS FAMILIES AND ANGER IN \\ ATHLETES AND PHYSICAL ACTIVITY \\ PRACTITIONERS
}

\author{
Higinio GONZÁLEZ GARCÍA ${ }^{1}$ Y \\ ANTONIA PELEGRÍN ${ }^{1}$
}

Cómo referenciar este artículo/How to reference this article:

González García, H. y Pelegrín, A. (2018). Familias numerosas e ira en deportistas y practicantes de actividad física [Numerous Families and Anger in Athletes and Physical Activity Practitioners]. Acción Psicológica, 15(1), 71-86. https://doi.org/10.5944/ap.15.1.21467

\section{Resumen}

Los objetivos fueron: (a) Conocer las diferencias en la ira en deportistas y practicantes de actividad física criados en familias numerosas y no numerosas; (b) Comprobar las diferencias en la ira en deportistas criados en familias numerosas y no numerosas; (c) Conocer las diferencias en la ira en deportistas criados en familias numerosas y no numerosas con éxitos nacionales. La muestra fue 442 deportistas y practicantes de actividad física. Se utilizó el Inventario de Expresión de Ira Estado-Rasgo (STAXI-II) y la Es- cala de Oviedo de Infrecuencia de Respuesta (INF-OV). Los resultados mostraron diferencias significativas en ira rasgo, temperamento, expresión interna e índice de expresión. Cuando se analizó al grupo de deportistas, excluyendo a los practicantes de actividad física, se encontraron diferencias significativas en ira rasgo, temperamento, expresión externa e índice de expresión. Por otra parte, los deportistas con éxitos nacionales mostraron diferencias significativas en la reacción de ira. Se concluyó que los deportistas y practicantes de actividad física criados en familias numerosas pueden poseer menores niveles de ira que los de familias no numerosas. Por lo tanto, el número

Correspondencia: Higinio González García. Facultad de Psicología. Universidad Miguel Hernández de Elche.

Email: higinio.gonzalez@goumh.es

ORCID: Higinio González García (http://orcid.org/0000-0002-9921-744X) y Antonia Pelegrín (https://orcid.org/00000003-0370-6079)

${ }^{1}$ Universidad Miguel Hernández de Elche, España.

Recibido: 1 de marzo de 2018.

Aceptado: 8 de mayo de 2018. 
de hermanos ha de ser tenido en cuenta como variable de riesgo en los deportistas que presentan altos niveles de esta emoción.

Palabras clave: Familia; Hijos; Emoción; Ira; Deporte.

\section{Abstract}

The objectives were: (a) To know the differences in anger in athletes and physical activity practitioners raised in large and non-numerous families; (b) Check the differences in anger in athletes raised in large families and not numerous; (c) Know the differences in anger in athletes raised in large families and not numerous with national successes. The sample consisted of 442 athletes and physical activity practitioners. To measure the different variables were used, the State-Trait Anger Expression Inventory (STAXI-II) and the Oviedo Scale of Infrequency of Response (INF-OV). The results showed significant differences in trait anger, temperament, internal anger expression and anger expression index. When the group of athletes was analysed separately, excluding the physical activity practitioners' group, the results showed significant differences in trait anger, temperament, external anger expression and anger expression index. On the other hand, athletes with national successes showed significant differences in anger reaction. It was concluded that athletes and physical activity practitioners grown up in large families have lower levels of anger than those of non-numerous families. Therefore, the number of siblings must be considered as a risk variable in athletes who have high levels of this emotion.

Keywords: Family; Children; Emotion; Sport.

\section{Introducción}

El número de hijos por familia ha ido disminuyendo en España paulatinamente durante las últimas dos décadas, siendo actualmente lo más prevalente las parejas que viven sin hijos (21\%) (INE, 2016). Siguiendo los datos del INE (2016) el mayor número de parejas tienen un hijo (47.87 \%), el segundo lugar lo ocupan las familias con dos hijos (45.75\%) y en último lugar las familias con más de tres hijos $(9.39 \%)$. Por lo tanto, la estructura familiar se muestra como una variable de interés en el estudio del comportamiento de los hijos y cómo se refleja esta influencia en el campo deportivo, ya que ha sido un tópico poco estudiado dentro del ámbito del deporte.

Además, diversos trabajos han probado la influencia del número de hijos en las conductas desviadas y en la socialización posterior (Cangas, Gázquez, Pérez, Moldes y Rubio, 2007; Piñero, Arense y Cerezo, 2013; Piñero, López-Espín, Cerezo y Torres-Cantero, 2012). De esta forma, en varios trabajos se encontró que las familias con un solo hijo presentaron mayores problemas de convivencia, mayores niveles de aislamiento e impopularidad (Cangas et al., 2007; Duncan, 1999; Piñero et al., 2012; Piñero et al., 2013). En otros estudios se ha mostrado que los hijos únicos pueden estar más sobreprotegidos por sus familias, más centrados en sí mismos y esto puede presentar un desajuste negativo en su desarrollo psicosocial (Doh, 1999; Kuepie, Tenikue, Nouetagni y Misangumukini, 2011; Sui, Wang, Liu y Wang, 2015). Al mismo tiempo, en otros trabajos se ha encontrado que el hecho de tener hermanos puede servir como factor protector dentro de la familia, siempre y cuando el número de hijos no sea muy numeroso (Eriksen y Jensen, 2006; Sui et al., 2015). Por otro lado, en otro trabajo se encontró que las familias numerosas pueden influir negativamente en la relación entre los padres, disminuyendo su satisfacción marital (Vera, FélixCastro y Rodríguez-Barreras, 2001). En este caso, siguiendo la Ley 40/2003, de 18 de noviembre, de protección a las familias numerosas, se entiende la familia numerosa como aquella que se compone de más de tres hijos en el hogar familiar, aunque hay otras excepciones que hacen que una familia sea numerosa con dos hijos como puede ser la presencia de discapacidad, orfandad, etc. Por otra parte, en un trabajo de Piñero et al. (2013) mostraron que en las familias con tres o más hermanos aumentaban los porcentajes de agresores en el campo escolar hasta un $28.07 \%$ y los de víctimas de acoso hasta el $24.56 \%$. Por lo tanto, el número de hijos se muestra como una variable susceptible de influir en el ajuste psicológico y en sus niveles de ira de los deportistas y practicantes de actividad 
física. Además, el ajuste psicológico es necesario para la práctica deportiva, debido a las cambiantes situaciones que se dan en el espectáculo deportivo (Brière et al., 2018).

En este sentido, la ira se ha mostrado como una variable de gran influencia en el ámbito deportivo y de la actividad física, tanto en el rendimiento deportivo, como en la elección deportiva, ajuste emocional, comportamientos agresivos, elección deportiva, estrategias metacognitivas, etc. (Davis, 2011; González-García, Pelegrín y Carballo, 2017; Jones, 2003; Lane, Beedie, Devonport y Stanley, 2011). Por todo ello, el estudio de la ira en el deporte y actividad física ha cobrado un gran interés por la comunidad científica durante la última década (Davis, 2011). Siguiendo a Spielberger, Jacobs, Rusell y Crane (1983, pp. 162), la ira se entiende como "un estado emocional caracterizado por sentimientos de enojo o de enfado y que tienen una intensidad variable". Además, la ira puede ser entendida como "rasgo" o "estado", rasgo si es la tendencia a experimentar ira de manera estable en el tiempo y estado cuando se experimenta ira de manera esporádica por un evento estresante (Spielberger, Miguel-Tobal, Casado y Cano-Vindel, 2001; Spielberger, Johnson, Russell, Crane, Jacobs y Worden, 1985). La ira posee un componente adaptativo con significado evolutivo, por lo que prepara para el ataque a las personas que poseen mayores niveles de esta emoción (Deffenbacher y McKay, 2000; Lench, 2004).

En este sentido, en el ámbito deportivo la ira puede interferir en el rendimiento a través de la interrupción o el aumento de la atención, el procesamiento de la información y la toma de decisiones, la ejecución y, finalmente, el control del rendimiento (Jones, 2003; Wittmann, Arce y Santiseban, 2008). Además, en los deportes de combate y contacto la ira puede actuar como un factor que aumenta el rendimiento deportivo y en aquellos deportes de alto control motor, en los que no existe el contacto, este exceso de energía que provoca la ira puede actuar como factor depresor del rendimiento (Hanin, 2007; Martinent y Ferrand, 2009). Por otro lado, cuando se relaciona a la ira con las estrategias de afrontamiento se encontró que los deportistas que obtuvieron mayor control de la ira mostraron mejores estrategias de afrontamiento ante los problemas, en comparación con los que obtuvieron resultados más bajos
(Bolgar, Janelle y Giacobbi, 2008). Por otra parte, la ira también ha mostrado relación con la creencia acerca de las metaemociones. Lane et al. (2011) mostraron que los deportistas que creían que la ansiedad o la ira eran positivas para su rendimiento deportivo obtuvieron diferencias significativas en la ira, pero no de ansiedad, antes de la competición. Además, la ira también ha mostrado relación con la elección deportiva (Maxwell, Visek y Moores, 2009). De manera que en un trabajo de Maxwell et al. (2009) se mostró que los deportistas con mayores niveles de ira tendieron a elegir los deportes de contacto e individuales. En otro trabajo de González-García et al. (2017), los deportistas con mayor expresión interna de la ira se relacionaron con la práctica de deporte de raqueta. Además, en varios trabajos se ha mostrado diferencias significativas en la ira a favor de los deportes colectivos (Baron y Richardson, 1994; Pelegrín, Serpa y Rosado, 2013). De otro modo, en el ámbito de actividad física y salud se ha demostrado que la actividad física ayuda a reducir los niveles de ira, mostrándose como una importante arma para reducir las emociones negativas y aumentar las positivas (Dubnov y Berry, 2013; Reigal y Videra, 2013).

Por otro lado, no se han encontrado trabajos anteriores que examinen el número de hijos del hogar familiar con los niveles de ira en deportistas. Por lo tanto, vista la influencia que tiene el número de hijos en el hogar familiar en otros campos diferentes al deporte y a la actividad física, como novedad de este trabajo, se muestra interesante conocer la influencia que muestra el número de hijos en el campo de la actividad físico-deportiva en adultos. De esta manera, con este trabajo se pretende aumentar el conocimiento de estas variables al ámbito deportivo y de la actividad física.

Por lo tanto, una vez se han puesto los presupuestos teóricos que sustentan este trabajo de investigación, después de mostrar la relación entre el número de hijos y las variables relacionadas con la ira, y de conocer la mostrada influencia de la ira en el ámbito del deporte y actividad física, se establecieron los siguientes objetivos de investigación: 1) Conocer las diferencias en los niveles de ira en deportistas y practicantes de actividad física criados en familias numerosas y familias no numerosas; 2) Comprobar las diferencias en los niveles de ira en deportistas criados en familias numerosas y familias no numerosas. 3) Cono- 
cer las diferencias en los niveles de ira en los deportistas que pertenecen a familias numerosas y familias no numerosas que poseen éxitos a nivel nacional.

La hipótesis que se estableció para el primer objetivo es que los deportistas y practicantes de actividad física criados en familias no numerosas presentaran diferencias significativas en los niveles de ira, mostrando menores niveles. Por otro lado, en el segundo objetivo se hipotetiza que los deportistas criados en familias no numerosas presentaran diferencias significativas en los niveles de ira, mostrando menores niveles. Por otra parte, en el tercer objetivo se hipotetiza que los deportistas con éxitos nacionales criados en familia numerosa obtendrán diferencias significativas en los niveles de ira, mostrando menores niveles.

\section{Método}

\section{Participantes}

La muestra total se compuso de 442 deportistas y practicantes de actividad física, de los cuales el $72.6 \%$ eran deportistas y el $27.4 \%$ eran practicantes de actividad física. De ellos 133 son mujeres (30.1\%) y 309 son hombres $(69.9 \%)$ con una edad comprendida entre los 18 y los 64 años $(M=26.73, S D=8.27)$. A continuación, se examinó a los deportistas federados de la muestra, es decir aquellos que están asociados a federaciones deportivas para practicar su deporte, de los cuales, 195 eran federados (44.1\%), 247 eran no federados (55.9\%), 46 eran deportistas profesionales $(10.4 \%)$ y 119 obtuvieron éxitos nacionales $(26.9 \%)$. La mayoría de deportistas $(72.6 \%)$ practicaban: fútbol $(9.67 \%)$, ciclismo $(9.38 \%)$, running $(9.38 \%)$, tenis de mesa $(9.38 \%)$ y otros deportes $(62.19 \%)$. La mayoría de practicantes de actividad física (27.4\%) realizaba: musculación o trabajo de fuerza en el gimnasio (69.30\%), actividades dirigidas (zumba, ciclo indoor, baile, etc; $27.72 \%$ ) y senderismo (2.97 \%). El número de participantes que eran hijos únicos fue del $9.3 \%$, con dos $64.7 \%$ y con tres o más del $26 \%$.

Como criterio de inclusión de la muestra, se seleccionó a deportistas y practicantes de actividad física mayores de
18 años para conocer si el tipo de familia en la que se criaron influye en sus niveles de ira actuales. La muestra se seleccionó mediante un muestreo no probabilístico (incidental), asegurando la diversidad de deportes que la componen, intentando recoger participantes de la mayor diversidad de zonas de España y asegurando las garantías para la recogida de la información.

\section{Instrumentos de evaluación}

Cuestionario Sociodemográfico ad hoc. Para evaluar los factores sociodemográficos y el número de hermanos, se creó un cuestionario de elaboración propia. Los ítems valoraron aspectos relacionados con: variables biológicas del deportista (sexo, edad, etc.) y variables sociodeportivas (deporte, éxitos deportivos, profesional o amateur, etc) “Eres profesional de tu deporte?" "El número de hermanos que tengo contándome yo mismo es de". En función de la respuesta a esta pregunta cuantitativa se realizaron ambos grupos, el grupo de familias no numerosas comprendía de uno a dos hijos y el de numerosas presentaba tres hijos o más. Esta división se realizó siguiendo la definición de familia numerosa y nuclear realizada por la Ley 40/2003, de 18 de noviembre, de protección a las familias numerosas, en el punto uno de su artículo dos, tan sólo teniendo en cuenta el número de hijos. Del total de 28 ítems, cinco evaluaban variables biológicas, cinco evaluaban variables laborales y académicas y 18 valoraban variables sociodeportivas. La mayoría de las preguntas eran de respuesta cerrada, de las cuales había preguntas tipo Likert, dicotómicas y politómicas.

Aquiescencia y participantes deshonestos. Se utilizó la escala de Oviedo de Infrecuencia de Respuesta (INF-OV; Fonseca-Pedrero, Lemos-Giráldez, Paino, Villazón-García y Muñiz, 2009), es una medida de autoinforme que está compuesta por 12 ítems que consta de una escala Likert con 5 posibilidades de respuesta ( $1=$ "Completamente en desacuerdo"; 5 = "Completamente de acuerdo"). El objetivo de esta escala es detectar a los participantes que responden de forma aleatoria, pseudoaleatoria o deshonesta. Al realizarse el cuestionario online, esta escala garantiza la fiabilidad de las respuestas de los participantes. Los participantes con más de 3 respuestas incorrectas en esta prueba fueron retirados de la muestra. En este estudio, se 
eliminaron 25 participantes que habían respondido de manera deshonesta al cuestionario.

Ira. Para la medición de la Ira Rasgo, Expresión Externa de Ira, Expresión Interna de Ira, Temperamento, Reacción de Ira, Control Interno de Ira, Control Externo de Ira y del Índice de Expresión de la Ira, se utilizó el Inventario de Expresión de la Ira Estado-Rasgo STAXI-2 (Spielberger et al., 2001). Se utilizó la escala de Ira rasgo, la cual incluye cinco sub-escalas (Rasgo de Ira, Expresión Externa de Ira, Expresión Interna de Ira, Control Externo de Ira y Control Interno de Ira), dos subescalas (Temperamento de Ira y Reacción de Ira) y el Índice de Expresión de la Ira (IEI) que ofrece una medida general de la expresión y control de la ira. Las escalas empleadas en este trabajo obtuvieron un coeficiente alfa de Cronbach de .74 en esta investigación, lo que indica niveles apropiados de fiabilidad. En los estudios de validación de la escala original, el instrumento obtuvo una correlación test-retest a los dos meses de .71 y un coeficiente alfa de Cronbach de .82 y un coeficiente alpha de Cronbach de .89 para la escala de IraEstado, de .82 para la de Ira-Rasgo, y de .69 para la de Expresión de Ira (Spielberger et al., 2001). Por otro lado, en un estudio de Oliva y Calleja (2010) en el que se valida la escala al ámbito deportivo se obtuvo un alfa de Cronbach de .81 en deportistas mexicanos, encontrándose un alfa de Cronbach de .88 en la escala de temperamento, .61 en la escala de reacción ante la frustración, .80 en la de reacción ante la desvalorización, 64 en la de expresión externa, .65 en la de expresión interna y .80 en la de control interno.

\section{Procedimiento}

En primer lugar, el estudio fue evaluado por el comité de ética de la Universidad Miguel Hernández. A continuación, se procedió a buscar participantes contactando con las federaciones deportivas españolas, con entrenadores, deportistas y practicantes de actividad física. Se contactó con clubes, deportistas y practicantes de actividad física de toda la geografía española, ya que el estudio se realizó en línea. Los deportistas que manifestaban su interés en participar a los investigadores enviaban un email para recibir el cuestionario de investigación. Una vez que los deportistas recibían la batería de pruebas, podían realizarla desde casa en cualquier momento de su tiempo libre a través de Google Forms. Las respuestas de los cuestionarios se alojaron en la base de datos de Google Drive. El anonimato de los participantes estaba garantizado y se animó a que contestaran lo más verazmente posible.

\section{Análisis de Datos}

Para el tratamiento estadístico de los datos se utilizó el programa SPSS 19.0. Para conocer las características de la muestra, se realizaron los estadísticos descriptivos de media, mínimo, máximo, frecuencia, porcentaje y desviación típica. Para verificar que la muestra seguía una distribución normal, se utilizó la prueba de Komolgorov-Smirnov y se encontró que la muestra seguía una distribución normal $(p>.05)$; por lo tanto, se utilizaron pruebas paramétricas. Para conocer las diferencias de medias cuando las variables eran cuantitativas, se utilizó la prueba $t$ de student para muestras independientes, empleándose un nivel de confianza del $95 \%$. Para analizar el tamaño del efecto y así conocer la magnitud de las diferencias encontradas en la prueba $t$ de student, se utilizó la $d$ de Cohen. En este caso, siguiendo a Cohen (1988) se pueden considerar los resultados del tamaño del efecto de la siguiente forma: $d=.20$ (pequeño), $d=.50$ (moderado) y $d=.80$ (grande).

\section{Resultados}

En primer lugar, con el objetivo de conocer las diferencias en los niveles de ira en los deportistas y practicantes de actividad física que pertenecieron a familias numerosas y no numerosas, se realizó una prueba $t$ para muestras independientes, en la que subdividió a la muestra en Familias Numerosas (FN) y Familias No Numerosas (FNN). 
Tabla 1

Diferencias de medias en las variables de ira en las familias numerosas y no numerosas

\begin{tabular}{|c|c|c|c|c|c|c|c|c|}
\hline \multirow[t]{2}{*}{ Variables de Ira } & \multicolumn{2}{|c|}{$\begin{array}{c}\text { FNN } \\
(n=327)\end{array}$} & \multicolumn{2}{|c|}{$\begin{array}{c}\text { FN } \\
(n=115)\end{array}$} & \multirow[t]{2}{*}{$t$} & \multirow[t]{2}{*}{ (p) } & \multirow[t]{2}{*}{$g l$} & \multirow[t]{2}{*}{ d de Cohen } \\
\hline & $\mathrm{M}$ & $(D T)$ & $\mathrm{M}$ & $(D T)$ & & & & \\
\hline Ira Rasgo & 22.69 & $(5.32)$ & 21.31 & $(4.68)$ & 2.46 & $(.014)^{*}$ & 440 & .23 \\
\hline Temperamento & 8.96 & $(3.16)$ & 8.12 & (2.70) & 2.56 & $(.011)^{*}$ & 440 & .24 \\
\hline Reacción de Ira & 13.72 & (3.25) & 13.19 & (3.02) & 1.53 & $(.120)$ & 440 & \\
\hline Expresión Externa Ira & 11.71 & (2.96) & 11.17 & $(2.71)$ & 1.73 & $(.080)$ & 440 & \\
\hline Expresión Interna Ira & 14.10 & (3.59) & 13.31 & (3.56) & 2.03 & $(.043)^{*}$ & 440 & .19 \\
\hline Control Externo Ira & 19.47 & (3.34) & 19.86 & (3.30) & -1.07 & $(.280)$ & 440 & \\
\hline Control Interno Ira & 16.23 & (4.19) & 16.43 & $(3.76)$ & -0.45 & $(.640)$ & 440 & \\
\hline Índice de Expresión de Ira & 26.11 & $(8.55)$ & 24.19 & $(7.92)$ & 2.11 & $(.035)^{*}$ & 440 & .20 \\
\hline
\end{tabular}

Nota. FNN = Familia No Numerosa, FN = Familia Numerosa.

${ }^{*} p<.05 ;{ }^{* *} p<.01$.

En la Tabla 1, los resultados mostraron diferencias significativas en ira rasgo $(p<.05)$, temperamento $(p<.01)$, expresión interna de ira $(p<.01)$ e índice de expresión de ira $(p<.05)$ en aquellos deportistas y practicantes de actividad física de familias no numerosas.

En segundo lugar, con el objetivo de conocer las diferencias en los niveles de ira en los deportistas que pertenecen a familias numerosas y familias no numerosas, se realizó una prueba $t$ para muestras independientes. En este caso se seleccionó de la muestra a los practicantes de deporte $(n=321)$, excluyendo a los practicantes de actividad física $(n=123)$.
En la Tabla 2, los resultados mostraron diferencias significativas en ira rasgo $(p<.01)$, temperamento $(p<.01)$, expresión externa de ira $(p<.05)$ e índice de expresión de ira $(p<.05)$, en los deportistas de familias no numerosas.

En tercer lugar, con el objetivo de conocer las diferencias en los niveles de ira en los deportistas que pertenecen a familias numerosas y familias no numerosas que poseen éxitos a nivel nacional, se realizó una prueba $t$ para muestras independientes. En este caso se seleccionó de la muestra a los deportistas con éxitos a nivel nacional $(n=119)$.

Tabla 2

Diferencias de medias en las variables de ira en función del número de hermanos en deportistas

\begin{tabular}{|c|c|c|c|c|c|c|c|c|}
\hline \multirow[t]{2}{*}{ Variables de Ira } & \multicolumn{2}{|c|}{$\begin{array}{c}\text { FNN } \\
(n=81)\end{array}$} & \multicolumn{2}{|c|}{$\begin{array}{c}\text { FN } \\
(n=240)\end{array}$} & \multirow[t]{2}{*}{$t$} & \multirow[t]{2}{*}{ (p) } & \multirow[t]{2}{*}{$g l$} & \multirow[t]{2}{*}{ d de Cohen } \\
\hline & $\mathrm{M}$ & $(D T)$ & $\mathrm{M}$ & $(D T)$ & & & & \\
\hline Ira Rasgo & 22.93 & $(5.02)$ & 20.72 & $(4.30)$ & 2.720 & $(.007)^{\star *}$ & 319 & .39 \\
\hline Temperamento & 9.12 & (3.26) & 7.70 & $(2.54)$ & 2.750 & $(.006)^{\star *}$ & 319 & .39 \\
\hline Reacción de Ira & 13.80 & (3.02) & 13.02 & (2.73) & 1.580 & $(.110)$ & 319 & \\
\hline Expresión Externa Ira & 11.87 & (3.02) & 10.72 & (2.86) & 2.310 & $(.022)^{*}$ & 319 & .33 \\
\hline Expresión Interna Ira & 14.35 & (3.63) & 13.25 & (3.72) & 1.810 & $(.070)$ & 319 & \\
\hline Control Externo Ira & 19.14 & (3.38) & 19.81 & (3.68) & -1.160 & $(.240)$ & 319 & \\
\hline Control Interno Ira & 16.14 & (4.23) & 16.14 & (3.88) & 0.006 & $(.240)$ & 319 & \\
\hline Índice de Expresión de Ira & 26.93 & $(8.62)$ & 24.02 & $(9.06)$ & 2.010 & $(.046)^{*}$ & 319 & .28 \\
\hline
\end{tabular}

Nota. FNN = Familia No Numerosa, FN = Familia Numerosa.

${ }^{*} p<.05 ;{ }^{* *} p<.01$. 
Tabla 3

Diferencias de medias en las variables de ira en función de la obtención de éxitos a nivel nacional

\begin{tabular}{|c|c|c|c|c|c|c|c|c|}
\hline \multirow[t]{2}{*}{ Variables de Ira } & \multicolumn{2}{|c|}{$\begin{array}{c}\text { FNN } \\
(n=94)\end{array}$} & \multicolumn{2}{|c|}{$\begin{array}{c}\text { FN } \\
(n=25)\end{array}$} & \multirow[t]{2}{*}{$t$} & \multirow[t]{2}{*}{ (p) } & \multirow[t]{2}{*}{$g l$} & \multirow[t]{2}{*}{ d de Cohen } \\
\hline & $\mathrm{M}$ & $(D T)$ & $\mathrm{M}$ & $(D T)$ & & & & \\
\hline Ira Rasgo & 22.62 & (5.25) & 20.56 & $(4.92)$ & 1.77 & $(.07)$ & 117 & \\
\hline Temperamento & 8.82 & (3.06) & 8.16 & $(2.73)$ & 0.99 & $(.32)$ & 117 & \\
\hline Reacción de Ira & 13.79 & (3.20) & 12.40 & $(2.78)$ & 1.98 & $(.04)^{*}$ & 117 & .65 \\
\hline Expresión Externa Ira & 11.52 & (2.73) & 10.76 & (2.72) & 1.23 & (.21) & 117 & \\
\hline Expresión Interna Ira & 14.28 & (3.53) & 13.04 & (3.91) & 1.53 & (.12) & 117 & \\
\hline Control Externo Ira & 19.88 & (3.44) & 19.96 & (3.25) & -0.10 & (.92) & 117 & \\
\hline Control Interno Ira & 16.56 & $(4.06)$ & 16.20 & (4.70) & 0.38 & $(.70)$ & 117 & \\
\hline Índice de Expresión de Ira & 25.36 & $(7.61)$ & 23.64 & $(8.81)$ & 0.97 & $(.33)$ & 117 & \\
\hline
\end{tabular}

Nota. FNN = Familia No Numerosa, FN = Familia Numerosa.

${ }^{*} p<.05 ;{ }^{* *} p<.01$.

En la Tabla 3, los resultados mostraron diferencias significativas en la reacción de ira $(p<.05)$, a favor de los deportistas con éxitos nacionales criados en familias numerosas.

\section{Discusión}

Los objetivos de este trabajo fueron: 1) Conocer las diferencias en los niveles de ira en deportistas y practicantes de actividad física criados en familias numerosas y familias no numerosas; 2) Comprobar las diferencias en los niveles de ira en deportistas criados en familias numerosas y familias no numerosas; 3) Conocer las diferencias en los niveles de ira en los deportistas que pertenecen a familias numerosas y familias no numerosas que poseen éxitos a nivel nacional. En relación al primer y segundo objetivo, los resultados mostraron diferencias significativas en ira rasgo, temperamento, expresión interna de ira e índice de expresión de ira, en aquellos deportistas y practicantes de actividad física de familias no numerosas. Cuando se analizó por separado al grupo de deportistas, los resultados mostraron diferencias significativas en ira rasgo, temperamento, expresión externa de ira e índice de expresión de ira, en los deportistas de familias no numerosas. Estos resultados siguen los hallazgos de los estudios precedentes en otros campos de la vida distintos al deporte, en los que se encuentra que los hijos únicos presentan peores niveles de socialización y mayores de agresión (Ann, 1990; Doh, 1999; Sui, Wang, Liu y Wang, 2015; Tucker y Finkelhor,
2015). Aunque en este trabajo científico dentro del grupo de familias no numerosas se incluyó a las familias con hijos únicos y familias con dos hijos. Al igual que argumentan los estudios previos que hablan de las familias (Ann, 1990; Doh, 1999; Cangas et al., 2007; Duncan, 1999; Piñero et al., 2013; Piñero et al., 2012; Sehan, 2016; Sui et al., 2015), las familias no numerosas pueden tener hijos más centrados en sí mismos, con mayores problemas de socialización, más egocéntricos, menos maduros y esto puede influir en que presenten mayores niveles de ira. Además, al tener menos hermanos sus conductas sociales están menos estimuladas dentro del hogar, lo que puede influir en que tengan menos habilidades sociales, menos conductas de empatía y menos conductas de habilidades interpersonales, lo que puede hacer que aumenten sus niveles de ira (Cangas et al., 2007; Duncan, 1999; Piñero et al., 2013; Piñero et al., 2012). A su vez, al vivir con menos personas en el hogar, puede que estén acostumbrados a conseguir todo lo que quieren y esto haga que tengan menor tolerancia a la frustración, lo que también puede aumentar los niveles de ira (Cangas et al., 2007; Duncan, 1999). Por lo tanto, se confirma la primera y segunda hipótesis establecida, los deportistas y el grupo de deportistas y practicantes de actividad física de familias no numerosas poseen mayores niveles de ira que los de las numerosas. Por otra parte, destaca que el grupo de deportistas muestra diferencias en la expresión de su ira de manera externa; y cuando se mide a deportistas y practicantes de actividad física juntos, la expresión externa no mostró diferencias significativas, sino la interna. Un estudio de Al- 
tin (2015) muestra que el deporte puede mediatizar esta expresión externa de la ira, la cual está considerada como más sana a nivel de salud.

En relación al tercer objetivo, conocer las diferencias en los niveles de ira en los deportistas que pertenecen a familias numerosas y familias no numerosas que poseen éxitos a nivel nacional. Los resultados mostraron diferencias significativas en los niveles de reacción de ira, en los deportistas con éxitos nacionales de familias numerosas que obtuvieron menores niveles. Estos resultados siguen en concordancia con los estudios previos mostrados anteriormente (Ann, 1990; Doh, 1999; Sehan, 2016; Sui et al., 2016) que muestran en otros campos diferentes al deporte que las familias numerosas presentan un efecto psicosocial positivo en los hijos. Además, existen otros trabajos que muestran que el nivel de rendimiento deportivo también influye en los niveles de ira (Menéndez-Santurio y Fernández-Río, 2015; Woodman, Davis, Hardy, Callow, Glasscock y Yuill-Proctor, 2009); por ello, el nivel de rendimiento deportivo puede que se muestre como un factor mediador a que no se encuentren diferencias en las otras variables que se encontraron anteriormente (ira rasgo, temperamento, expresión interna de ira e índice de expresión de ira). Por lo tanto, este resultado confirma la tercera hipótesis establecida, y muestra que dentro de los deportistas con éxitos nacionales la familia numerosa puede influir en la reacción de ira de estos deportistas.

Como líneas de propuesta futura, sería interesante conocer cómo se relaciona el número de hijos con las conductas prosociales y con la ira, por la importante implicación que han mostrado las conductas prosociales en la ira y para conocer si hay diferencias en la socialización en función del número de hijos de cada familia. Además, sería interesante conocer si el orden de los hermanos muestra relación con poseer mayores niveles de ira, ya que sería una variable de interés para estudiar en el deporte. A su vez, en futuros estudios sería de interés conocer las diferencias entre las familias con un hijo, dos hijos y numerosas, ya que en este trabajo científico, al ser el primer trabajo de esta temática, tan sólo se dividieron en no numerosas y numerosas. De esta manera podría conocerse las diferencias interindividuales entre los tres grupos de familias. Al mismo tiempo, también sería interesante conocer cómo influye en los niveles de ira el aumento en el número de hijos. Por otro lado, los resultados de este trabajo muestran que en el ámbito deportivo un factor de riesgo que puede ser importante en los niveles de ira es pertenecer a una familia no numerosa, ello debe ser tomado en cuenta por psicólogos deportivos y entrenadores cuando se trabaja el control de esta emoción en los deportistas.

Entre las limitaciones principales de este trabajo de investigación destaca la agrupación en familias numerosas y no numerosas, ya que puede haber diferencias entre el número de hijos. Por lo tanto, dentro de esta clasificación podría subdividirse en más clasificaciones y no sólo en estas dos. De este modo, se conocería cómo evolucionan los resultados según el número de hijos. Además, pueden existir más variables que estén mediando las diferencias en los niveles de ira de los deportistas y practicantes de actividad física de familias no numerosas y numerosas. Por lo tanto, la dificultad de medir otras variables que pueden mediar estos resultados se muestra como una debilidad dentro de este trabajo.

Como conclusiones, los deportistas y practicantes de actividad física criados en familias numerosas pueden poseer menores niveles de ira que los de familias no numerosas. Por lo tanto, en los perfiles de deportistas más irascibles el número de hermanos debe ser una variable a tener en cuenta, por las posibilidades que tiene de influir en los niveles de ira del deportista. Por otro lado, los deportistas con éxitos a nivel nacional criados en familias numerosas poseen menores niveles de reacción de ira que los deportistas con éxitos nacionales criados en familias no numerosas. Por lo tanto, el tipo de familia también se muestra como una variable que influye en los niveles de ira de los deportistas con éxitos nacionales.

Como aplicaciones prácticas, el número de hermanos ha de ser tenido en cuenta por los profesionales de la psicología deportiva como variable de riesgo en los deportistas y practicantes de actividad física que presentan altos niveles de la emoción de ira. Asimismo, en aquellos deportistas y practicantes de actividad física de familias no numerosas los profesionales de la psicología deben fortalecer el trabajo del control emocional desde el ámbito familiar, para así de este modo poder prevenir problemas de gestión emocional en el ámbito deportivo. 


\section{Referencias}

Altin, M. (2015). Anger Expression Styles of Hearing Impaired Individuals Doing Sport and those not Doing Sport. Educational Research and Reviews, 10(17),

2406-2412. https://doi.org/10.5897/err2015.2430

Ann, L. (1990). Only Children in Britain: Popular Stereotype and Research Evidence. Children and Society, 4, 386-400. https://doi.org/10.1111/j.10990860.1990.tb00375.x

Baron, R. A. y Richardson, D. (1994). Human Aggression. New York: Plenum Press.

Bolgar, M. R., Janelle, C. y Giacobbi, P. R. (2008). Trait Anger, Appraisal and Coping Differences among Adolescent Tennis Players. Journal of Applied Sport Psychology, 20(1), 73-87. https://doi.org/10.1080/10413200701790566

Brière, F. N., Yale-Soulière, G., Gonzalez-Sicilia, D., Harbec, M. J., Morizot, J., Janosz, M. y Pagani, L. S. (2018). Prospective Associations between Sport Participation and Psychological Adjustment in Adolescents. Journal of Epidemiology Community Health, 14, 209-656. https://doi.org/10.1136/jech2017-209656

Cangas, A. J., Gázquez, J. J., Pérez-Fuentes, M. C., Moldes, P. y Rubio, C. (2007). Influencia de las características familiares en la percepción de los Conflictos de Convivencia Escolar [Influence of Family Characteristics on the Perception of Conflicts of School Coexistence]. Típica: Boletín Electrónico de Salud Escolar, 3(1). Recuperado de http://repositorio.ual.es/bitstream/handle/10835/204 7/TIPICA.pdf?sequence=1\&isAllowed=y

Cohen, J. (1988). Statistical Power Analysis for the Behavioral Sciences. New York: Academic Press.

Davis, P. A. (2011). Angry Athletes: Psychological, Physiological, and Performance Implications. En J. P. Welty (Ed.), Psychology of Anger: Symptoms,
Causes and Coping (pp. 197-212). New York, NY: Nova Science.

Deffenbacher, J. L. y Mckay, M. (2000). Overcoming Situational and General Anger. A Protocol for the Treatment of Anger Based on Relaxation, Cognitive Restructuring, and Coping Skills Training. Therapist protocol. Oakland: New Harbinger.

Doh, H. F. T. (1999). Social Competence, Maternal Attentiveness, and Overprotectiveness: Only Children in Korea. International Journal of Behavioural Development, 23, 149-162.

Dubnov, G. y Berry, E. M. (2013). Physical Activity and Mood. The Endocrine Connection. Humana Press, Totowa, NJ.

Duncan, R. (1999). Peer and Sibling Aggression: An Investigation of intra- and extra-Familial Bullying. Journal of Interpersonal Violence, 14(8), 871-886. https://doi.org/10.1177/088626099014008005

Eriksen, S. J. y Jensen, V. (2006). All in the Family? Family Environment Factors in Sibling Violence. Journal of Family Violence, 21(8), 497-507. https://doi.org/10.1007/s10896-006-9048-9

Fonseca-Pedrero, E., Lemos-Giráldez, S., Paino, M., Villazón-García, U. y Muñiz, J. (2009). Validation of the Schizotypal Personality Questionnarie Brief form in adolescents. Schizophrenia Research, 111(1-3), 53-60.

González-García, H., Pelegrín., A. y Carballo, J. L. (2017). Ira y personalidad resistente en deportistas de raqueta y resistencia [Anger and Hardy Personality in Racket and endurance sportsmand]. Revista Iberoamericana de Ciencias de la Actividad Física y el Deporte, 6(2), 21-29. Recuperado de http://www.revistas.uma.es/index.php/riccafd/article /view/3776

Sehan, C. L. (2016). The Wiley Blackwell Encyclopedia of Family Studies. New Jersey: John Wiley. 
Hanin, Y. (2007). Emotions in Sports: Currents Issues and Perspectives. En G. Tenembaum y R. Eklund (Eds.), Handbook of Sport Psychology (pp. 31-58). New Jersey: Wiley.

INE. (2016). Instituto Nacional de Estadística. Nota continua de hogares [Statistics National Institute. Continuous note of households]. Recuperado de http://www.ine.es/prensa/np965.pdf

Jones, M. V. (2003). Controlling Emotions. The Sport Psychologist, 17(4), https://doi.org/10.1123/tsp.17.4.471

Kuepie, M., Tenikue, M., Nouetagni, S. y Misangumukini, N. (2011). Number of Siblings and School Achievement in sub Sahara Africa. Luxembourg: CEPS, instead (Working Paper No 2011-31).

Lane, A. M., Beedie, C. J., Devonport, T. J. y Stanley, D. M. (2011). Instrumental Emotion Regulation in Sport: Relationships between Beliefs about Emotion and Emotion Regulation Strategies Used by Athletes. Scandinavian Journal of Medicine and Science in Sports, 21(6), 445-451. https://doi.org/10.1111/j.1600-0838.2011.01364.x

Lench, H. C. (2004). Anger Management: Diagnostic Differences and Treatment Implications. Journal of Social and Clinical Psychology, 23(4), 512-531. https://doi.org/10.1521/jscp.23.4.512.40304

Ley 40/2003, de 18 de noviembre, de protección a las familias numerosas. Boletín Oficial del Estado, núm. 277, de 19 de noviembre de 2003, pp. 1 a 14 . https://www.boe.es/buscar/pdf/2003/BOE-A-200321052-consolidado.pdf

Maxwell, J. P., Visek, A. J. y Moores, E. (2009). Anger and Perceived Legitimacy of Aggression in Male Hong Kong Chinese Athletes: Effects of Type of Sport and Level of Competition. Psycholy Sport and Exercise, $\quad 10, \quad 289-296$. https://doi.org/10.1016/j.psychsport.2008.07.010
Martinent, G. y Ferrand, C. (2009). A Naturalistic Qualitative Study of the Directional Interpretation Process of Discrete Emotions during High-Stakes Table Tennis Matches. Journal of Sport \& Exercise Psychology, 31, 318-336.

Menéndez-Santurio, J. I. y Fernández-Río, J. (2015). Niveles de ira en practicantes de boxeo y kickboxing: diferencias en función de la disciplina y el nivel deportivo [Anger Intensity in Boxing and Kickboxing Practitioners: Differences Based on Discipline and Competitive Level]. Cuadernos de Psicología del Deporte, 15(3), 75-86. Recuperado de http://revistas.um.es/cpd/article/view/244481

Oliva, F. J. y Calleja, N. (2010). Medición de la ira en el deporte de combate: Validación del STAXI-2 en deportistas mexicanos [Measurement of Anger in Combat Sport: Validation of STAXI-2 in Mexican Sportsmen]. Liberabit: Lima, 16(1), 51-60. Recuperado de http://www.scielo.org.pe/scielo.php?script=sci_artte xt\&pid=S1729-48272010000100006

Oliva, F., Calleja, N. y Hernández-Pozo, R. (2012). Escala de creencias sobre la ira en el deporte de combate con atletas mexicanos [Cale of Beliefs about Anger in Combat Sports Among Mexican Athletes]. Revista Internacional de Medicina y Ciencias de la Actividad Física y el Deporte, 12(45), 110-121. Recuperado de https://repositorio.uam.es/handle/10486/8749

Pelegrín, A., Serpa, S. y Rosado, A. (2013). Aggressive and Unsportsmanlike Behaviours in Competitive Sports: An Analysis of Related Personal and Environmental Variables. Anales de Psicología, 29(3), 701-713. http://dx.doi.org/10.6018/analesps.29.3.175841

Piñero, E., Arense, J. J. y Cerezo, F. (2013). Contexto familiar y conductas de agresión y victimización entre escolares de educación secundaria [Family Context and Aggression and Victimization in High School Students]. Bordón Revista de Pedagogía, 65(3), 109-129. https://doi.org/10.13042/23167 
Piñero, E., López-Espín, J. J., Cerezo, F. y Torres-Cantero, A. M. (2012). Tamaño de la fratría y victimización escolar [Number of Siblings and School Victimization]. Anales de Psicología, 28(3), 842847. https://doi.org/10.6018/analesps.28.3.156091

Reigal, R. y Videra, A. (2013). Effect of a Physical Activity Session on Mood States. Revista Internacional de Medicina y Ciencias de la Actividad Fisica y del Deporte 13(52), 783-798.

Spielberger, C. D., Miguel Tobal, J. J., Casado, M. I. y Cano Vindel, A. (2001). Inventario de Expresión de Ira Estado Rasgo 2-STAXI 2 [State Trait Expression of Anger Inventory 2-STAXI2]. Madrid, España: TEA.

Spielberger, C. D., Jacobs, G., Rusell, S. F. y Crane, R. S. (1983). Assessment of Anger: The State-Trait Anger Scale. En J. N. Butcher y C. D. Spielberger (Eds.), Advances in personality assessment (vol. 2, pp. 159-187). Hillsdale, N. J.: Lawrence Erlbaum Associates.

Spielberger, C. D., Johnson, E. H., Russell, S. F., Crane, R. J., Jacobs, G. A. y Worden, T. J. (1985). The Experience and Expression of Anger: Construction and Validation of an Anger Expression Scale. En M. A. Chesney y R. H. Rosenman (Eds.,) Anger and hostility in cardiovascular and behavioural disorders (pp. 5-30). Nueva York: Hemiphere/McGraw-Hill.
Sui, G. Y., Wang, J. N., Liu, G. C. y Wang, L. (2015). The Effects of Being an Only Child, Family Cohesion, and Family Conflict on Behavioural Problems among Adolescents with Physically Ill Parents. International Journal of Environmental Reearc and Public Health, 12(9), 10910- 10922. https://doi.org/10.3390/ijerph120910910

Tucker, C. J. y Finkelhor, D. (2015). The State of Interventions for Sibling Conflict and Aggression: A Systematic Review. Trauma, Violence and Abuse, 18(4), 396-406. https://doi.org/10.1177/1524838015622438

Vera, J., Félix-Castro, J. y Rodríguez-Barreras, A. (2001). Satisfacción marital, edad, número de hijos y años en pareja [Marital satisfaction, age, number of children and couple years]. Revista de Psicología, Universidad Mayor de San Marcos, 1-2, 233-250.

Wittmann, M., Arce, E. y Santisban, C. (2008). How Impulsiveness, Trait Anger and extra Circular Activities Might Affect Higher Aggression in School Children. Personality and Individual Differences, 45(7), 618-623. https://doi.org/10.1016/j.paid.2008.07.001

Woodman, T., Davis, P. A., Hardy, L., Callow, N., Glasscock, I. y Yuill-Proctor, J. (2009). Emotions and Sport Performance: An Exploration of Happiness, Hope, and Anger. Journal of Sport and Exercise Psychology, 31(2), 169-188. 


\title{
NUMEROUS FAMILIES AND ANGER IN ATHLETES AND PHYSICAL ACTIVITY PRACTITIONERS
}

\author{
Higinio GONZÁLEZ GARCÍA ${ }^{1}$ Y \\ ANTONIA PELEGRÍN ${ }^{1}$
}

\section{EXTENDED SUMMARY}

\section{Introduction}

The number of children per family has decreased in Spain gradually for the last two decades, the most prevalent are couples with a single-child (47.87\%). In this way, several studies found that families with single-child had greater problems of coexistence, greater overprotection, higher levels of isolation and unpopularity (Cangas et al., 2007; Doh, 1999; Duncan, 1999; Kuepie et al., 2011; Piñero et al., 2013; Piñero et al., 2012; Sui et al., 2015). Furthermore, other studies have found that having siblings can become a protective factor within the family, if the number of children is not very numerous (Eriksen \& Jensen, 2006; Sui et al., 2015).

On the other hand, anger has been shown as a variable with great influence on sports and physical activity, sport performance, sport choice, emotional adjustment, aggressive behaviours, metacognitive strategies, etc (Davis, 2011; González-García et al., 2017; Jones, 2003; Lane et al., 2011). For all these reasons, the study of anger in sports and physical activity has gained great interest in the scientific community for the last decade (Davis, 2011).

In addition, there are not previous works that examine the family number of children with anger levels in athletes. Therefore, once the theoretical assumptions have been established, the objectives were: 1) To know the differences in anger levels in athletes and physical activity practitioners raised in large families and not numerous families; 2 ) Check the differences in anger levels in athletes raised in large families and non-numerous families. 3) To know the differences in anger levels in athletes belonging to large families and non-numerous families with sport national successes.

The hypothesis that was established for the first objective is that athletes and physical activity practitioners raised in non-numerous families will present significant differences in anger levels, showing lower levels. On the other hand, in the second goal it is hypothesized that athletes raised in non-numerous families will present significant differences in anger levels, showing lower levels. On the other hand, in the third aim it is hypothesized that athletes with national successes raised in large families will obtain significant differences in anger levels, showing lower levels.

\section{Method}

\section{Participants}

The sample was made up by 442 athletes and physical activity practitioners, $72.6 \%$ were athletes and $27.4 \%$ were physical activity practitioners. The gender of the sample was 133 women $(30.1 \%)$ and 309 men $(69.9 \%)$ with an age range from 18 to 64 years old $(M=26.73$, $S D=8.27)$. In the sample, 195 athletes were federated $(44.1 \%), 247$ were non-federated (55.9\%), 46 were professional $(10.4 \%)$ and 119 obtained sport national successes $(26.9 \%)$.

\section{Instruments}

Sociodemographic questionnaire ad hoc. To evaluate sociodemographic factors and the number of siblings, a 
self-created questionnaire was carried out. The items assessed aspects related to: the athlete's biological variables (sex, age, etc.) and socio-sporting variables (sports, sporting success, professional or amateur, etc.), e.g: "The number of siblings that I have counting myself is". Depending on the answer to this quantitative question, both groups were made, the group of non-numerous families comprised from one to two children and the numerous groups had three or more children.

Acquiescence and dishonest participants. The Oviedo Scale of Infrequency of Response (INF-OV; FonsecaPedrero et al., 2009) was used, it is made up by 12 items in a Likert scale with five response possibilities. The objective of this scale is to detect the participants who respond in a random, pseudo-random or dishonest way. Participants with more than three incorrect answers were removed from the sample. In this study, 25 participants who had responded dishonestly were deleted.

Anger. For the Measurement of Anger Trait, External Anger Expression, Internal Anger Expression, Temperament, Anger Reaction, Internal Anger Control, External Anger Control and the Anger Expression Index, the Anger State-Trait Expression Inventory (STAXI-2; Spielberger et al., 2001) was used.

\section{Procedure}

Firstly, the ethics committee of the Miguel Hernández University evaluated the study. The athletes who expressed their interest in participating sent an email to the researchers to receive the survey. The responses to the questionnaires were stored in the Google Drive database. The anonymity of the participants was guaranteed, and they were encouraged to answer as truthfully as possible.

\section{Data analysis}

For the statistical treatment of the data, SPSS 19.0 program was used. To verify that the sample followed a normal distribution, the Komolgorov-Smirnov test was conducted and the sample followed a normal distribution ( $p>.05)$. In order to know the mean differences when the variables were quantitative, the student's $t$ test for inde- pendent samples was performed, using a confidence level of $95 \%$. Furthermore, the effect size was analysed with the Cohen $d$.

\section{Results}

Table 1, the results showed significant differences in trait anger $(p<.05)$, temperament $(p<.01)$, internal anger expression $(p<.01)$ and anger expression index $(p<.05)$, in those athletes and physical activity practitioners from non-numerous families.

Table 2, the results showed significant differences in trait anger $(p<.01)$, temperament $(p<.01)$, external anger expression $(p<.05)$ and anger expression index $(p<.05)$, in the athletes from not numerous families.

Table 3 , the results showed significant differences in anger reaction $(p<.05)$, in favour of athletes with national successes raised in large families.

\section{Discussion}

The objectives of this work were: 1) To know the differences in anger levels in athletes and physical activity practitioners raised in large families and not numerous families; 2) To check the differences in anger levels in athletes raised in large families and non-numerous families; 3) To know the differences in anger levels in athletes belonging to large families and non-numerous families with national sport successes. Concerning the first and second goal, the results showed significant differences in trait anger, temperament, internal anger expression and anger expression index, in benefit of those athletes and physical activity practitioners from non-numerous families. When the group of athletes was analysed separately, the results showed significant differences in trait anger, temperament, external anger expression and anger expression index in athletes from non-numerous families. These results follow the previous findings in other fields different than sport, where it was found that single children have worse levels of socialization and greater levels of aggression 
(Ann, 1990; Doh, 1999; Sui et al., 2015; Tucker \& Finkelhor, 2015).

Although, in this work the group of non-numerous families included families with single-children and families with two children. As previous studies presented (Ann, 1990; Doh, 1999; Cangas et al., 2007; Duncan, 1999; Piñero et al., 2013; Piñero et al., 2012; Sehan, 2016; Sui et al., 2015), non-numerous families may have more self-centred children, with greater socialization problems, less maturity and this may influence them to have higher levels of anger. In addition, having fewer siblings may influence them to have less social skills, less empathy and lack of social skills, which can increase their anger levels (Cangas et al., 2007; Duncan, 1999; Piñero et al., 2013; Piñero et al., 2012). Furthermore, single-child live with fewer people at home, which may accustom them to get everything they want, and this will make them less adaptive to frustration tolerance and increase their anger levels (Cangas et al., 2007; Duncan, 1999). Therefore, the first and second hypothesis established were confirmed, the athletes and the group of athletes and physical activity practitioners of non-numerous families have greater anger levels than those from the numerous. On the other hand, the group of athletes indicated differences in external anger expression; and when it was measured athletes and physical activity practitioners together, the external expression did not show significant differences, but only the internal anger expression. Consequently, a research study by Altin (2015) showed that sports can mediate this external anger expression, which is considered to be healthier.

Concerning the third aim, to know the differences in anger levels in athletes that belong to large families and not numerous families that have sport national successes. The results showed significant differences in anger reaction levels, in athletes with national successes from numerous families that obtained lower levels. These results are consistent with previous studies (Ann, 1990; Doh, 1999; Sehan, 2016; Sui et al., 2016) that showed in other fields different from sports that numerous families have a positive psychosocial effect in children. In addition, there are other works which presented that sport performance level is also influenced by anger levels (Menéndez-Santurio \& Fernández-Río, 2015; Woodman et al., 2009); therefore, the athletic performance level may be shown as a me- diating factor to do not find differences in other variables that were previously found (trait anger, temperament, internal anger expression and anger expression index). Therefore, this result confirms the third established hypothesis, and shows that among athletes with national successes the large family can influence the anger reaction.

As future proposal lines, it would be interesting to know how is related the number of children to prosocial behavior and anger, the important implication that prosocial behaviors have shown in anger and to know if there are differences in socialization depending on the children number of each family. In addition, it would be interesting to know if the siblings birth order, shows a relationship with having higher anger levels. On the other hand, the results of this work show that in the sports field a risk factor that can be important in anger levels is to belong to a non-numerous family, it must be considered by sports psychologists and coaches, in order to work the control of this emotion in athletes.

The main limitations of this research work are: grouping into large and non-numerous families, because there may be differences between the children number. Thus, this classification could be subdivided into more classifications and not only in these two. In this regard, we would know how the results evolve according to the number of children. In addition, there may be more variables that are mediating the differences in athletes anger levels and physical activity practitioners from numerous and not numerous families. Therefore, the difficulty of measuring other variables that can mediate these results is shown as a weakness in this work.

As conclusions, athletes and physical activity practitioners raised in large families may have lower anger levels than those from non-numerous families. Therefore, in more anger athletes profiles the number of siblings should be a variable to be considered, because of the possibilities it has to influence the athlete's anger levels. On the other hand, athletes with national success raised in large families have lower levels of anger reaction than athletes with national successes raised in not numerous families. Therefore, the family type is also shown as a variable that influences the anger level of athletes with national successes. 
As practical applications, the number of siblings must be considered by sports psychology professionals as a risk variable in athletes and physical activity practitioners who have high levels of anger emotion. Even though, in those athletes and physical activity practitioners of non-numerous families, professionals in psychology must strengthen the work of emotional control from the family environment, in order to prevent emotional management problems in sport. 
